\title{
Assessment of Fine Structure Processing Strategies in Unilaterally Deafened Cochlear Implant Users
}

\author{
Dayse Távora-Vieira',2, Gunesh P. Rajan"1,2 \\ ${ }^{1}$ Otolaryngology, Head \& Neck Surgery, School of Surgery, University of Western Australia, Perth, Australia \\ ${ }^{2}$ Fremantle Hospital, Fremantle, Australia \\ Email: dayse.tavora@health.wa.gov.au
}

Received 6 September 2014; revised 5 October 2014; accepted 3 November 2014

Academic Editor: Roberta Marino, University of Western Australia, Australia

Copyright (C) 2014 by authors and Scientific Research Publishing Inc.

This work is licensed under the Creative Commons Attribution International License (CC BY).

http://creativecommons.org/licenses/by/4.0/

(c) (i) Open Access

\section{Abstract}

This study aimed to investigate the speech perception and subjective preference of unilaterally deafened cochlear implant users for two different speech coding strategies. Thirteen subjects who received a cochlear implant were provided with 2 maps that differed in the speech coding strategy, FS4 or FS4-p (MED-EL). Subjects were requested to alternate between the two maps daily for two weeks and to complete a questionnaire daily. Speech perception testing was performed using the adaptive Bamford-Kowal-Bench speech-in-noise test (BKB-SIN) after two weeks of alternating FS4/FS4-p use. The subjective benefit of FS4-p was significantly greater than the subjective benefit of FS4 on all five questions of the questionnaire. There was a significant improvement in speech perception scores over time under the $S_{0} / \mathrm{N}_{0}, S_{0} / \mathrm{N}_{\mathrm{HE}}, \mathrm{S}_{\mathrm{CI}} / \mathrm{N}_{\mathrm{HE}}$ test conditions. There was no significant difference between the speech perception scores obtained with FS4 and FS4-p coding strategies. For this group of cochlear implant recipients, assessment of the subjective preference for the speech coding strategy is likely to enhance motivation, compliance and consequently, outcomes.

\section{Keywords}

CI, Unilateral Deafness, FS4, FS4-p

\section{Introduction}

The technology and surgical techniques for auditory implants have advanced rapidly in recent years enabling us to treat various types and degrees of hearing loss. In addition, there is a continuous interest in improving signal 
processing and speech coding strategies. The improvements are designed not only to improve cochlear implant (CI) users' speech understanding in quiet and in noise, but also to enhance appreciation of music, and speech understanding in tonal languages.

Various speech coding strategies have been developed, all of which aim to provide CI users with the clearest and most natural sound possible given the constraints of the limited stimulation representation of the implant electrodes. Envelope representation of an incoming sound signal is a common theme in many speech-encoding strategies, examples of which include Continuous Interleaved Sampling (CIS) [1], HiResolution (HiRes) [2], and Advanced Combination Encoder (ACE) [3]. One of MED-EL's (Innsbruck, Austria) coding strategies, called Fine Structure Processing (FSP) [4], uses envelope representation (High Definition-CIS) and low frequency temporal information to improve subtle pitch discrimination and temporal cues in the low frequencies [5] (reviewed by Moore et al. [6]). There are reports of a general subjective preference for FSP over CIS+ coding strategies as well as a better appreciation of music using FSP [7]. The presentation of the fine structure is thought to enhance CI users' music appreciation, and speech understanding in noise [8]. Arnoldner et al. [9] reported that speech and music perception was improved with FSP when compared to CIS in the early stage of the study, but this improvement was not statistically significant at the 12-month follow up [10]. FS4 and FS4-p, developments of the FSP coding strategy, both have fine structure information delivered to designated low-frequency apical channels which can span $70 \mathrm{~Hz}-950 \mathrm{~Hz}$. While FS4 can stimulate just one low-frequency fine structure channel at any point in time, FS4-p can simultaneously stimulate two of the four fine structure channels at any given time and can thus provide the temporal code specific to each of the two channels with higher accuracy. Recently, Riss et al. [11] compared FS4 and FS4-p with FSP in terms of speech perception, sound quality and subjective preference. It was found that there was no significant difference among the three strategies for speech performance in noise. At the end of the study, 20 out 33 participants chose FS4 or FS4-p over FSP.

In the last few years, several studies have investigated the benefits of cochlear implantation in individuals with unilateral deafness (UD). There is a growing literature demonstrating that cochlear implantation decreases tinnitus disturbance associated with UD, improves speech understanding in noise, enhances localization ability and improves patients' self-perception of hearing performance [12]-[19].

Unilaterally deafened subjects commonly expect to match the hearing from the CI to their normal hearing in the contralateral ear. However, to best of our knowledge, there are no studies that address whether unilaterally deafened CI users demand different mapping techniques or modification of map parameters.

Unilaterally deafened CI users are in the unique position of being able to assess and compare the quality of speech coding strategies directly with the correlating sound percepts of their normal hearing ear. In this study, it was proposed that subjective evaluation of sound quality, and ease/effort of listening should also be explored, as the speech perception tests in isolation are insufficient to address these dimensions of hearing. Therefore, the present study sets out to evaluate how unilaterally deafened CI users subjectively perceive and rate sound when using two different speech coding strategies. It aimed to investigate if the differences between FS4 and FS4-p had a subjective benefit for unilaterally deaf cochlear implant users. We expected subtle differences between FS4 and FS4-p, and therefore formulated an open questionnaire that aimed to obtain information regarding the subjective perception.

\section{Material and Methods}

Thirteen adult subjects (7 males, 6 females) with post-lingual UD who received a MED-EL implant were recruited for this study. The mean age at implantation was 56 years (range 39 - 74). Further demographic data is presented in Table 1. The better hearing ear had a pure tone average $\left(\mathrm{PTA}_{0.5-4 \mathrm{kHz}}\right)$ of $\leq 32 \mathrm{~dB}$ and the ear to be implanted had a $\left(\mathrm{PTA}_{0.5-4 \mathrm{kHz}}\right)$ of $\geq 72 \mathrm{~dB}$. A hearing aid was fitted to the poorer ear if any functional hearing was present, and if this was unsuccessful, a patient was considered for a CI. Prior to implantation, all patients were offered a two week trial of both a conventional contra-lateral routing of signal amplification (CROS) hearing aid and a bone anchored hearing aid (Baha) mounted on a headband. Standardized pre-operative evaluation of the subjects included high-resolution computed tomography (CT) and magnetic resonance imaging (MRI) of the temporal bones and brain to rule out the presence of any inner ear anomalies or cochlear nerve pathologies that might constrain electrical stimulation by a CI. The audiological evaluation consisted of immitance measures, audiometry and speech discrimination in quiet using Arthur Boothroyd (AB) words [20].

Speech perception testing was performed using the adaptive Bamford-Kowal-Bench speech-in-noise test 
Table 1. Subject demographic data.

\begin{tabular}{cccccc}
\hline Subject & $\begin{array}{c}\text { Duration of deafness } \\
\text { (years) }\end{array}$ & $\begin{array}{c}\text { Age at implantation } \\
\text { (years) }\end{array}$ & Ear & $\begin{array}{c}\text { Pure tone average } \\
(0.5,1,2 \text { and } 4 \mathrm{kHz}) \text { - } \\
\text { non implanted ear in } \mathrm{dB}^{*}\end{array}$ & $\begin{array}{c}\text { Pure tone average } \\
(0.5,1,2 \text { and } 4 \mathrm{kHz})- \\
\text { implanted ear in } \mathrm{dB}^{*}\end{array}$ \\
\hline 1 & 1.5 & 73 & $\mathrm{~L}$ & 21 & $>110$ \\
2 & 1 & 57 & $\mathrm{~L}$ & 25 & 76 \\
3 & 5 & 71 & $\mathrm{R}$ & 30 & 74 \\
4 & 4 & 39 & $\mathrm{R}$ & 19 & $>110$ \\
5 & 2.5 & 74 & $\mathrm{~L}$ & 21 & 95 \\
6 & 7 & 53 & $\mathrm{R}$ & 24 & 80 \\
7 & 1 & 57 & $\mathrm{~L}$ & 32 & 92 \\
8 & 0.5 & 42 & $\mathrm{~L}$ & 10 & 87 \\
9 & 1.5 & 47 & $\mathrm{R}$ & 21 & 90 \\
10 & 1.0 & 53 & $\mathrm{~L}$ & 18 & 77 \\
11 & 3.0 & 76 & $\mathrm{R}$ & 27 & $>110$ \\
13 & 1.5 & 39 & $\mathrm{R}$ & 13 & 86
\end{tabular}

PTA = pure tone average at 0.5, 1, 2 and 4 kHz. R = right; L = left. *Before cochlear implantation.

(BKB-SIN) [21] which investigates the signal to noise ratio needed to achieve 50\% speech perception. Tests were performed in a free-field with the subject seated 1 meter away from loudspeakers located at angles of 0 , -90 and +90 degrees. The following spatial configurations were used: $\mathrm{S}_{0} / \mathrm{N}_{0}$-speech and noise presented from the front; $\mathrm{S}_{0} / \mathrm{N}_{\mathrm{HE}}$-speech presented from the front and noise to the normal hearing ear; and $\mathrm{S}_{\mathrm{CI}} / \mathrm{N}_{\mathrm{HE}}{ }^{-} \mathrm{Speech}$ presented to the implanted ear and noise to the side of the normal hearing ear.

All subjects were implanted with a FLEX ${ }^{\text {SOFT }}$ electrode (MED-EL, Austria) and received an OPUS 2 speech processor. Subjects were fitted with their speech processors 2 weeks after cochlear implantation. Mapping sessions took place weekly for the first four weeks. All patients had 3 months of CI experience prior to the experiment, and were using the FSP speech coding strategy since CI activation.

Three months post-CI activation, after giving written consent to participate in this study, subjects were provided with 2 maps called program 1 (P1) and program 2 (P2) that differed only in the coding strategy, FS4 or FS4-p, respectively. The subjects were blind to the different settings P1 and P2, and were not provided with any information regarding the differences between the two programs. The subjects were requested to alternate between the two maps daily for two consecutive weeks and to complete a non-standardized 10 point scale questionnaire at the end of each day, and return it to the audiologist. The patients' scores were averaged for each question for each program (P1 and P2). The questionnaire comprised of 5 questions:

1) How similar is the sound from the cochlear implant to the other ear? In this question the scale varied from 1 = "very different" to 10 = "very similar".

2) How is the clarity of sounds? The scale varied from 1 = "very unclear" to 10 = "very clear".

3) How easy is it to hear in quiet? The scale varied from 1 = "very difficult" to 10 = "very easy".

4) How easy is it to hear in noise? The scale varied from 1 = "very difficult" to 10 = "very easy".

5) How do you like the sound? The scale varied from $1=$ "not at all" to 10 = "very much".

Speech perception in noise test was performed at the end of the two weeks. The sequence of the test (P1/P2) was randomized. The audiologist performing the test was blind to the settings of the speech processor.

This study was designed and conducted in accordance with the Declaration of Helsinki, and ethical approval was obtained from the relevant ethics and institutional review committees.

Wilcoxon signed-rank test was used to determine the difference between the coding strategies for all 5 questions and for the speech perception in noise scores. A repeated measure ANOVA was performed to see if there was a significant improvement from preoperative to postoperative speech perception scores on the 3 spatial conditions.

A p-value of $<0.05$ was considered statistically significant. IBM SPSS Statistics 19 (IBM, Armonik, New 
York) software was used for the data analyses. Graphs were created using Microsoft Office Excel 2010 (http://www.microsoft.com).

\section{Results}

Figure 1 illustrates the difference between FS4 and FS4-p for each question presented to the unilaterally deafened CI users. The speech coding strategy FS4-p scored significantly higher than FS4 for all 5 questions. The results are shown in Table 2.

There was a significant improvement across all test intervals in the BKB-SIN under the $\mathrm{S}_{0} / \mathrm{N}_{0}$ test condition ( $\mathrm{p}<$ 0.001) (Figure 2). The improvement in the BKB-SIN under the $\mathrm{S}_{0} / \mathrm{N}_{0}$ test condition was significant between pre-operative testing and the $\mathrm{P} 1$ program test $(\mathrm{p}=0.001)$; and significant between pre-operative testing and the $\mathrm{P} 2$ program test $(\mathrm{p}=0.001)$. There was no significant difference between P1 and P2 in the BKB-SIN under the $\mathrm{S}_{0} / \mathrm{N}_{0}$ test condition $(\mathrm{p}=1.000)$.

There was a significant improvement across all test intervals in the BKB-SIN in the $\mathrm{S}_{0} / \mathrm{N}_{\mathrm{HE}}$ test condition $(\mathrm{p}=$ 0.002) (Figure 2). The improvement in the BKB-SIN under the $\mathrm{S}_{0} / \mathrm{N}_{\mathrm{HE}}$ test condition was significant between pre-operative testing and the P1 program test $(\mathrm{p}=0.005)$; and significant between pre-operative testing and the $\mathrm{P} 2$ program test $(\mathrm{p}=0.007)$. There was no significant difference between the P1 and P2 in the BKB-SIN under the $\mathrm{S}_{0} / \mathrm{N}_{\mathrm{HE}}$ test condition $(\mathrm{p}=0.763)$.

There was a significant improvement across all test intervals in the BKB-SIN in the $\mathrm{S}_{\mathrm{CI}} / \mathrm{N}_{\mathrm{HE}}$ test condition ( $\mathrm{p}<$ 0.001) (Figure 2). The improvement in the BKB-SIN under the $\mathrm{S}_{\mathrm{CI}} / \mathrm{N}_{\mathrm{HE}}$ test condition was significant between pre-operative testing and the $\mathrm{P} 1$ program test $(\mathrm{p}=0.002)$; and significant between pre-operative testing and the $\mathrm{P} 2$ program test $(\mathrm{p}=0.002)$. There was no significant difference between the P1 and P2 in the BKB-SIN under the $\mathrm{S}_{\mathrm{CI}} / \mathrm{N}_{\mathrm{HE}}$ test condition ( $\left.\mathrm{p}=0.157\right)$. The majority of the patients (10 out of 13) kept either FS4 or FS4-p at the end of the study.

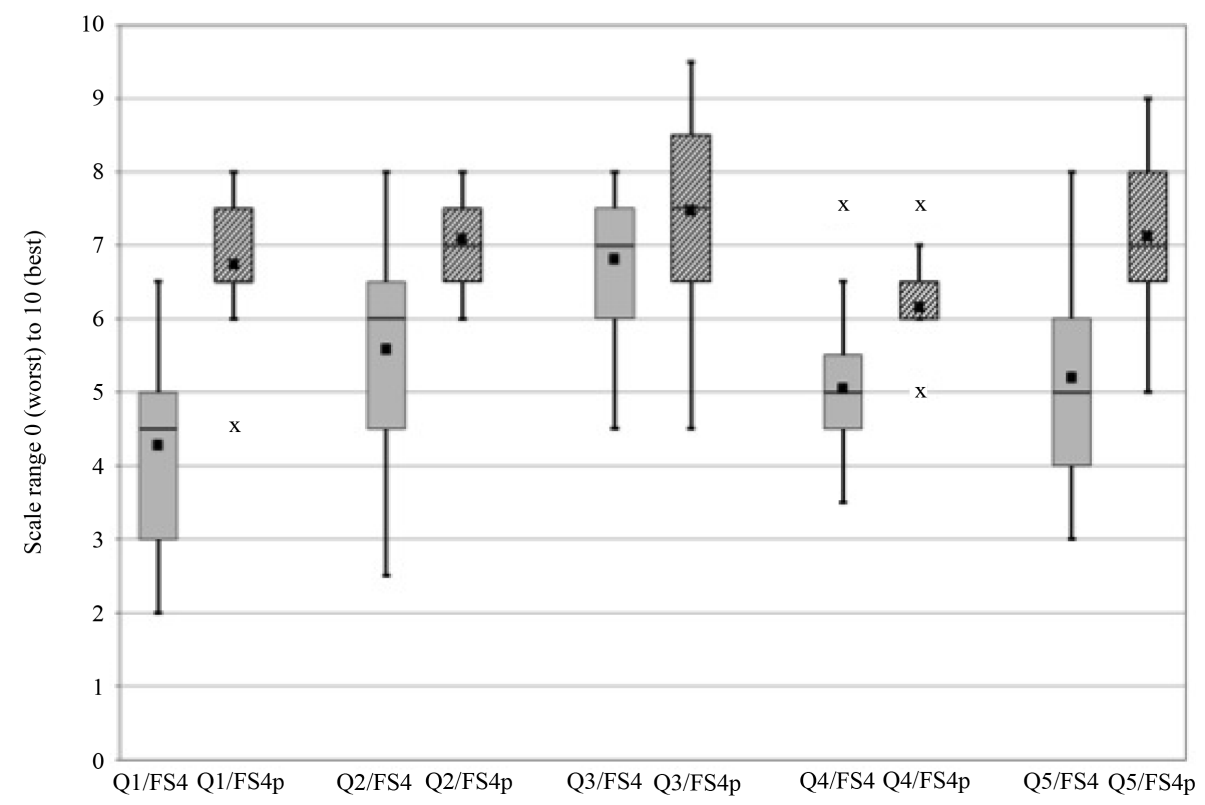

Figure 1. The group results for each of the five questions. FS4 is shown in grey boxes. FS4-p is shown in diagonally lined boxes. Mean values are depicted as black squares, median as horizontal lines, and asterisks are the outliers (calculated as 1.5 to 3 times box height above the 75th percentile).

Table 2. Wilcoxon signed-rank test results for questions 1-5.

\begin{tabular}{cccccc} 
& Q1 & Q2 & Q3 & Q4 & Q5 \\
\hline Z & -3.195 & -2.632 & -1.906 & -2.562 & -2.874 \\
p-value (2-sided) & 0.001 & 0.008 & 0.057 & 0.010 & 0.004 \\
\hline
\end{tabular}


BKB SIN speech perception scores

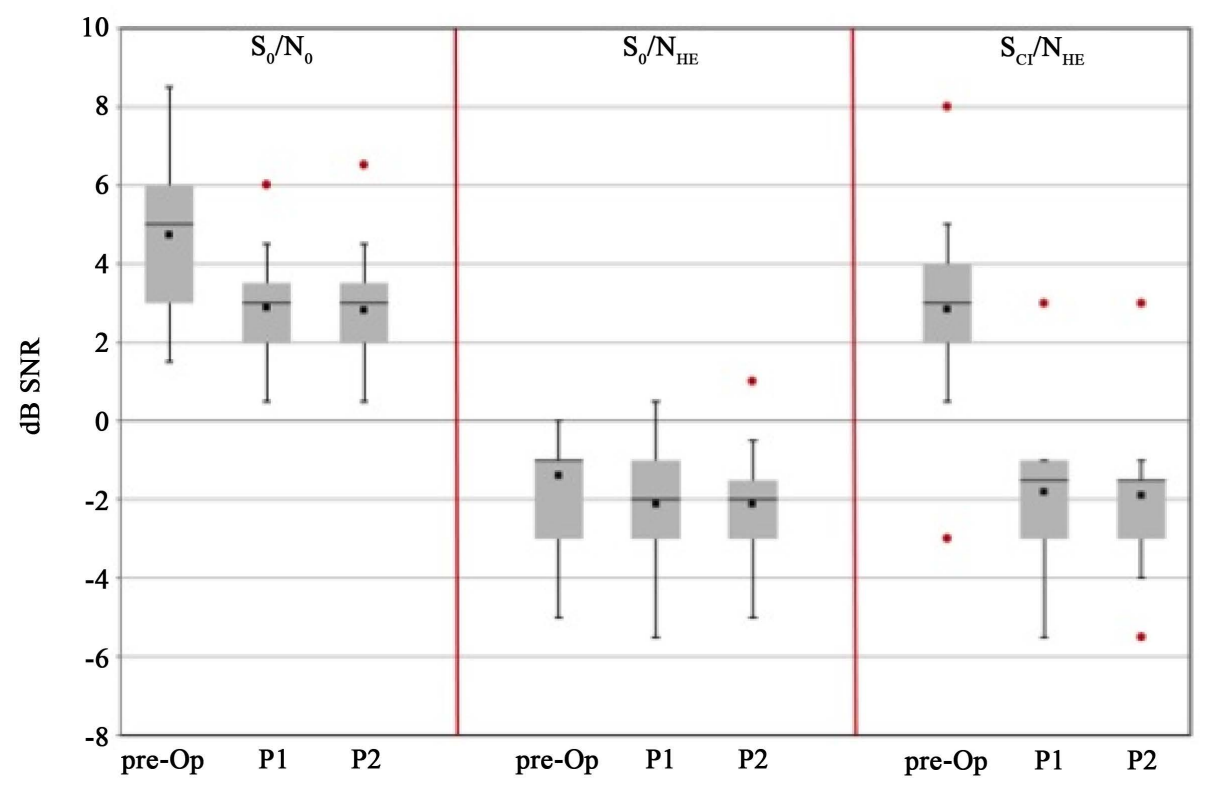

Figure 2. Results of the Bamford-Kowal-Bench speech-in-noise $(n=13)$ with speech presented from the front and noise presented from the front $\left(\mathrm{S}_{0} / \mathrm{N}_{0}\right)$; speech presented from the front and noise presented from the side of the normal hearing ear $\left(\mathrm{S}_{0} / \mathrm{N}_{\mathrm{HE}}\right)$, and; speech presented from the side of the cochlear implant and noise presented from the side of the normal hearing ear $\left(\mathrm{S}_{\mathrm{CI}} / \mathrm{N}_{\mathrm{HE}}\right)$. Mean values are depicted as black squares, median values as horizontal lines, and dots signify outliers (1.5 to $3 \times$ box height above the 75 th percentile).

\section{Discussion}

Several studies have demonstrated that cochlear implantation is a suitable hearing rehabilitation option for adults with unilateral profound deafness. Among the hearing devices used in the rehabilitation of UD subjects, CI is the only option that provides ear specific information and thus potentially the benefits of binaural hearing. The studies have investigated the effects of cochlear implantation on tinnitus, subjective perception of improvement, speech understanding in noise and localization ability [12]-[19]. A review of the literature by Vlastarakos et al. [22] has emphasized that self-assessment questionnaires were commonly used to assess the patients' perception of improvement in daily listening conditions. In fact, Stelzig et al. [16] reported that the subjective rating of outcomes tended to be more positive than the objective measures which could be related to an inadequacy of speech perception test for the unilaterally deafened CI users.

To date, there is no literature that addresses whether the patients with unilateral deafness demand any different mapping strategies or mapping parameters. This study addressed this issue by comparing the speech coding strategies FS4 and FS4-p in terms of patients' performance in the adaptive speech in noise test and the patients' responses to a non-standardized questionnaire. The questionnaire was developed by the authors and aimed to determine the subjective perception of sound from the CI.

The results showed a significant improvement in speech perception in noise scores when speech and noise are presented from the front $\left(\mathrm{S}_{0} / \mathrm{N}_{0}\right)$, and when speech is presented from the front $\left(\mathrm{S}_{0} / \mathrm{N}_{\mathrm{HE}}\right)$, or from the CI side $\left(\mathrm{S}_{\mathrm{CI}} / \mathrm{N}_{\mathrm{HE}}\right)$ with the noise presented to the normal hearing ear. This was true for both speech coding strategies. Riss et al. [11] found that there was no significant difference among the three strategies FSP, FS4 and FS4-p using an adaptive sentence test in noise. Similarly, in this study, there was no significant difference between FS4 and FS4-p in the speech understanding measures. These finding were expected as the difference between FS4 and FS4-p is subtle.

Outcome performance studies (which investigated the superiority of one speech coding strategy over another) have predominantly focused on speech perception, for which objective speech perception testing is appropriate. Unilaterally deafened CI users are in the unique position of being able to assess and compare the quality of speech coding strategies directly with the correlating sound percepts of their normal hearing ear. Therefore, it 
was proposed that subjective evaluation of sound quality and ease/effort of listening should be added to the evaluation protocol with the final objective to facilitate patients' acclimatization to electrical stimulation.

The results indicated that FS4-p was rated significantly superior to FS4 in all five questions answered by the unilaterally deafened CI users. As per question 1, it appears that unilaterally deafened CI users perceived that FS4-p mimicked the sound quality of the normal hearing contralateral ear significantly better than FS4. This was reinforced by the rating in question 5 , since the patients reported to like the sound provided by the CI more when using FS4-p. The explanation for these results is not clear. As the patients alternated between the two strategies daily, they had the same experience with both settings and thus it unlikely that the patients have acclimatized to one setting in particular. To avoid any bias, the audiologist performing the speech in noise test was blind to which speech coding strategy was being used.

The FSP strategy with its fine structure coding [23] aims to improve pitch perception, which is thought to improve speech discrimination, sound localization, and music appreciation [7]. The original FSP strategy provides fine structure processing in 1 - 3 apical channels up to $470 \mathrm{~Hz}$. The newer developed FS4 and FS4-p provide it to the 4 most apical low-frequency channels. FS4 stimulates the apical channels sequentially, while FS4-p simultaneously stimulates 2 of the 4 designated low frequency apical channels from $70-950 \mathrm{~Hz}$, and this is thought to further enhance temporal information. This may explain why CI users preferred FS4-p to FS4.

The subjective results in this study differ from those reported by Riss et al. [11] since our group of unilaterally deafened subjects rated FS4-p superior to FS4. This difference might be linked to the variability of the subjects between the studies. The subjects with UD might have rated the sound in comparison to normal acoustic hearing, while patients with bilateral hearing loss may rate the sound quality based solely on their auditory memory and/or an input from acoustic amplification on the contra-lateral ear.

The results of this study need to be interpreted with caution, as it used a non-validated questionnaire and a small number of subjects. However, it is the first to provide some insights about the mapping strategies to be considered for patients with unilateral deafness. Combined with speech perception testing, assessment of the patients' subjective preference for a specific speech coding strategy may assist in the rehabilitation program for unilateral deafness, enhancing patients' motivation and compliance with CI use.

\section{Conclusion}

There were no significant differences in the speech perception in noise scores between FS4 and FS4-p. The FS4-p fine structure was rated higher subjectively than FS4 in the present study. Subjective evaluation may assist in the rehabilitation program of unilaterally deafened CI users potentially enhancing motivation, compliance, and, consequently, outcomes.

\section{Acknowledgements}

The authors would kindly like to acknowledge E. A. for statistical analyses; I. G. A. and U. D. for editing a version of this manuscript.

\section{References}

[1] Wilson, B.S., Finley, C.C., Lawson, D.T., Wolford, R.D., Eddington, D.K. and Rabinowitz, W.M. (1991) Better Speech Recognition with Cochlear Implants. Nature, 352, 236-238. http://dx.doi.org/10.1038/352236a0

[2] Koch, D.B., Osberger, M.J., Segel, P. and Kessler, D.K. (2004) High Resolution and Conventional Sound Processing in the HiResolution Bionic Ear: Using Appropriate Outcome Measures to Assess Speech Recognition Ability. Audiology \& Neuro-Otology, 9, 214-223. http://dx.doi.org/10.1159/000078391

[3] Kiefer, J., Hohl, S., Sturzebecher, E., Pfennigdorff, T. and Gstoettner, W. (2001) Comparison of Speech Recognition with Different Speech Coding Strategies (SPEAK, CIS, and ACE) and Their Relationship to Telemetric Measures of Compound Action Potentials in the Nucleus CI 24M Cochlear Implant System. Audiology, 40, 32-42. http://dx.doi.org/10.3109/00206090109073098

[4] Zierhofer, C.M. (2003) Electrical Nerve Stimulation Based on Channel Specific Sampling Sequences. US Patent No. 6594525.

[5] Müller, J., Brill, S., Hagen, R., Moeltner, A., Brockmeier, S.J., Stark, T., Helbig, S., Maurer, J., Zahnert, T., Zierhofer, C., Nopp, P. and Anderson, I. (2012) Clinical Trial Results with the MED-EL Fine Structure Processing Coding Strategy in Experienced Cochlear Implant Users. ORL, 74, 185-198. http://dx.doi.org/10.1159/000337089 
[6] Moore, B.C. (2008) The Role of Temporal Fine Structure Processing in Pitch Perception, Masking, and Speech Perception for Normal-Hearing and Hearing-Impaired People. Journal of the Association for Research in Otolaryngology, 9, 399-406. http://dx.doi.org/10.1007/s10162-008-0143-x

[7] Lorens, A., Zgoda, M., Obrycka, A. and Skarzynski, H. (2010) Fine Structure Processing Improves Speech Perception as Well as Objective and Subjective Benefits in Pediatric MED-EL COMBI 40+ Users. International Journal of Pediatric Otorhinolaryngology, 74, 1372-1378. http://dx.doi.org/10.1016/j.ijporl.2010.09.005

[8] Vermiere, K., Punte, A.K. and Van de Heyning, P. (2010) Better Speech Recognition in Noise with Fine Structure Processing Coding Strategy. ORL, 72, 305-311. http://dx.doi.org/10.1159/000319748

[9] Arnoldner, C., Riss, D., Brunner, M., Durisin, M., Baumgartner, W.D. and Hamzavi, J.S. (2007) Speech and Music Perception with the New Fine Structure Speech Coding Strategy: Preliminary Results. Acta Oto-Laryngologica, 127, 1298-1303. http://dx.doi.org/10.1080/00016480701275261

[10] Riss, D., Arnoldner, C., Baumgartner, W.D., Kaider, A. and Hamzavi, J.S. (2008) A New Fine Structure Speech Coding Strategy: Speech Perception at a Reduced Number of Channels. Otology \& Neurotology, 29, 784-788. http://dx.doi.org/10.1097/MAO.0b013e31817fe00f

[11] Riss, D., Hamzavi, J.S., Blineder, M., Honeder, C., Ehrenreich, I., Kaider, A., Baumgartner, W.D., Gstoettner, W. and Arnoldner, C. (2014) FS4, FS4-p and FSP: A 4-Month Crossover Study of Three Fine Structure Sound-Coding Strategies. Ear \& Hearing. http://dx.doi.org/10.1097/AUD.0000000000000063

[12] Van de Heyning, P., Vermeire, K., Diebl, M., Nopp, P., Anderson, I. and De Ridder, D. (2008) Incapacitating Unilateral Tinnitus in Single-Sided Deafness Treated by Cochlear Implantation. Annals of Otology, Rhinology \& Laryngology, 117, 645-652. http://dx.doi.org/10.1177/000348940811700903

[13] Vermeire, K. and Van de Heyning, P. (2009) Binaural Hearing after Cochlear Implantation in Subjects with Unilateral Sensorineural Deafness and Tinnitus. Audiology \& Neuro-Otology, 14, 163-171. http://dx.doi.org/10.1159/000171478

[14] Buechner, A., Brendel, M., Lesinski-Schiedat, A., Wenzel, G., Frohne-Buechner, C., Jaeger, B. and Lenarz, T. (2010) Cochlear Implantation in Unilateral Deaf Subjects Associated with Ipsilateral Tinnitus. Otology \& Neurotology, 31, 1381-1385.

[15] Arndt, S., Aschendorff, A., Laszig, R., Beck, R., Schild, C., Kroeger, S., Ihorst, G. and Wesarg, T. (2010) Comparison of Pseudobinaural Hearing to Real Binaural Hearing Rehabilitation after Cochlear Implantation in Patients with Unilateral Deafness and Tinnitus. Otology \& Neurotology, 32, 39-47. http://dx.doi.org/10.1097/MAO.0b013e3181fcf271

[16] Stelzig, Y., Jacob, R. and Mueller, J. (2011) Preliminary Speech Recognition Results after Cochlear Implantation in Patients with Unilateral Hearing Loss: A Case Series. Journal of Medical Case Reports, 5, 343. http://dx.doi.org/10.1186/1752-1947-5-343

[17] Firszt, J.B., Holden, L.K., Reeder, R.M., Waltzman, S.B. and Arndt, S. (2012) Auditory Abilities after Cochlear Implantation in Adults with Unilateral Deafness: A Pilot Study. Otology \& Neurotology, 33, 1339-1346. http://dx.doi.org/10.1097/MAO.0b013e318268d52d

[18] Hansen, M.R., Gantz, B.J. and Dunn, C. (2013) Outcomes after Cochlear Implantation for Patients with Single-Sided Deafness, Including Those with Recalcitrant Meniere’s Disease. Otology \& Neurotology, 34, 1681-1687. http://dx.doi.org/10.1097/MAO.0000000000000102

[19] Távora-Vieira, D., Marino, R., Krishnaswamy, J., Kuthubutheen, J. and Rajan, G.P. (2013) Cochlear Implantation for Unilateral Deafness with and without Tinnitus: A Case Series. Laryngoscope, 123, 1251-1255. http://dx.doi.org/10.1002/lary.23764

[20] Boothroyd, A. (1968) Developments in Speech Audiometry. Sound, 2, 3-10.

[21] Bench, J., Kowal, A. and Bamford, J. (1979) The BKB (Bamford-Kowal-Bench) Sentence Lists for Partially-Hearing Children. British Journal of Audiology, 13, 108-112. http://dx.doi.org/10.3109/03005367909078884

[22] Vlastarakos, P.V., Nazos, K., Tavoulari, E.F. and Nikolopoulos, T.P. (2013) Cochlear Implantation for Single-Sided Deafness: The Outcomes. An Evidence-Based Approach. European Archives of Oto-Rhino-Laryngology, 271, 21192126. http://dx.doi.org/10.1007/s00405-013-2746-Z

[23] Schatzer, R., Krenmayr, A., Au, D.K. and Zierhofer, C. (2010) Temporal Fine Structure in Cochlear Implants: Preliminary Speech Perception Results in Cantonese Speaking Implant Users. Acta Oto-Laryngologica, 130, 1031-1039. http://dx.doi.org/10.3109/00016481003591731 
Scientific Research Publishing (SCIRP) is one of the largest Open Access journal publishers. It is currently publishing more than 200 open access, online, peer-reviewed journals covering a wide range of academic disciplines. SCIRP serves the worldwide academic communities and contributes to the progress and application of science with its publication.

Other selected journals from SCIRP are listed as below. Submit your manuscript to us via either submit@scirp.org or Online Submission Portal.
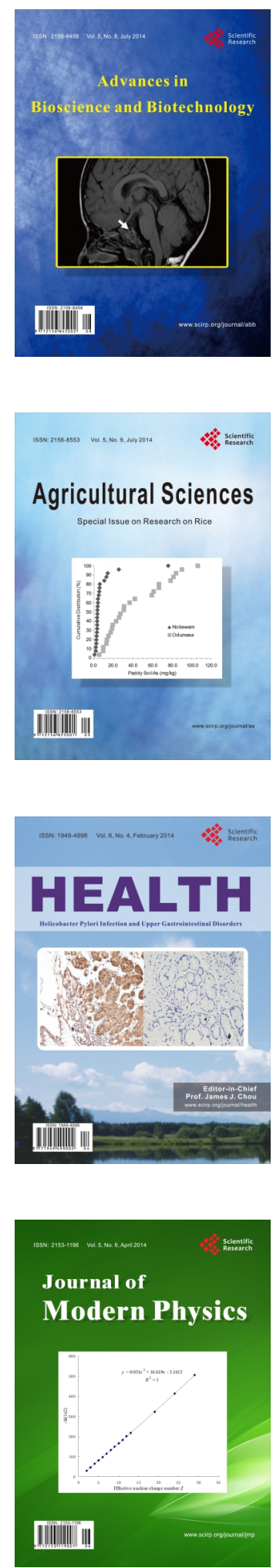
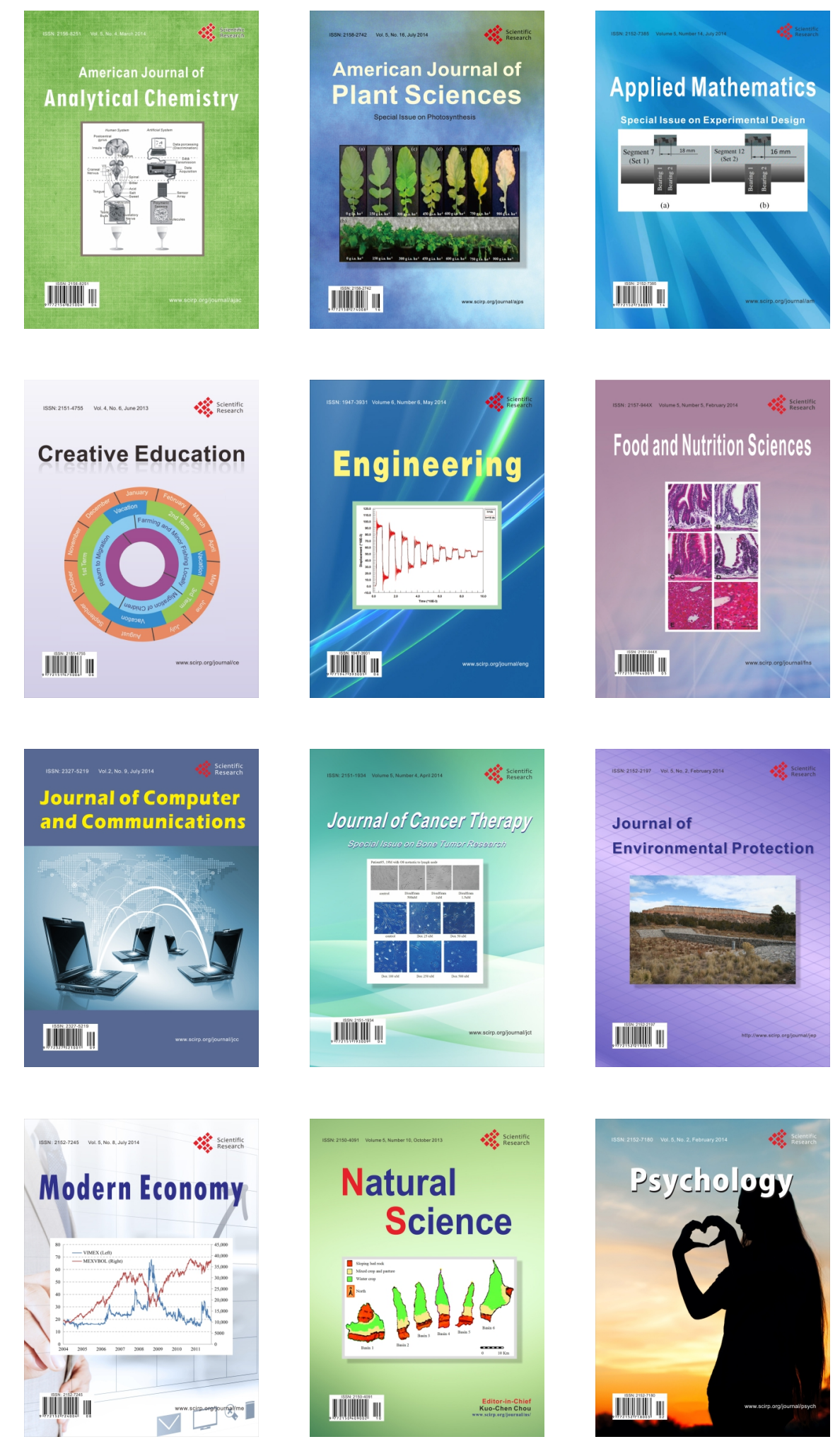